\title{
Studies of Ion Acoustic Decay
}

R. Paul Drake

Lawrence Livermore National Laboratory

Livermore, California

March 7, 1994

This is an informal report intended primarily for internal or limited external distribution. The opinions and conclusions stated are those of the author and may or may not be those of the Laboratory.

Work performed under the auspices of the U.S. Department of Energy by the Lawrence Livermore National Laboratory under Contract W-7405-Eng-48. 
This document was prepared as an account of work sponsored by an agency of the United States Government. Neither the United States Government nor the University of California nor any of their employees, makes any warranty, express or implied, or assumes any legal liability or responsibility for the accuracy, completeness, or usefulness of an information, apparatus, product, or process disclosed, or represents that its use would not infringe privately owned rights. Reference herein to any specific commercial products, process, or service by trade name, trademark, manufacturer, or otherwise, does not necessarily constitute or imply its endorsement, recommendation, or fa voring by the United States Government or the University of California. The views and opinions of authors expressed herein do not necessarily state or reflect those of the United States Government or the University of California, and shall not be used for advertising or product endorsement purposes.

This report has been reproduced directly from the best 2 vailable copy.

Available to DOE and DOE contractors from the Ofice of Scientific and Technical Information P.O. Box 62, O2k Ridge, TN 37831

Prices a vailable from (615) 576-8401, FTS 626-8401

Available to the public from the National Technical Information Service

U. 3. Department of Commerce 5285 Port Royal Rd., Springtield, VA 22161 
Concluding Report:

\section{Studies of Ion Acoustic Decay}

Principal Investigator:

R. Paul Drake

Tracking code $92-\mathrm{IN}-033$

Funded during FY92 and FY93

Collaborators:

B.S. Bauer, ${ }^{a}$ K.L. Baker, ${ }^{a, b}$ K. Mizuno, a,b B. Sleaford, ${ }^{a, b}$ A.V. Rubenchik, a, b

J.S. De Groot, ${ }^{b}$ Kent Estabrook, ${ }^{c}$ C. Labaune, ${ }^{d}$ S. Baton, ${ }^{d}$ T. Jalinaud, ${ }^{d}$ E. Schifano, ${ }^{d}$

M.V. Goldman, ${ }^{e}$ D.L. Newman, ${ }^{e}$ W. Seka, ${ }^{f}$ R. Bahr $f$

(a) Plasma Physics Research Institute, Lawrence Livermore National Laboratory

L-418, P.O.B. 808, Livermore CA 94551

(b) Department of Applied Science, University of California Davis, Davis CA 95616

c) X-division, Department of Physics, Lawrence Livermore National Laboratory and

University of California Davis, L-472 P.O.B. 808, Livermore CA 94551

(d) Laboratoire pour l'Utilisation des Lasers Intenses, Ecole Polytechnique,

Palaiseau 94100, France,

(e) Department of Astrophyscial, Planetary, and Atmospheric Sciences,

Campus Box 391, University of Colorado, Boulder CO 80309

(f) Laboratory for Laser Energetics, University of Rochester, 150 East River Road, Rochester, NY. 


\section{ABSTRACT}

In this project, we advanced knowledge of Ion Acoustic Decay on several fronts. In this project, we have developed and demonstrated the capability to perform experimental and theoretical studies of the Ion Acoustic Decay Instability. We have at the same time demonstrated an improved capability to do multichannel spectroscopy and Thomson scattering. We made the first observations of the time-resolved second harmonic emission at several angles simultaneously, and the first observations of the emission both parallel and perpendicular to the electric field of the laser light. We used Thomson scattering to make the first observations of the plasma waves driven by acoustic decay in a warm plasma with long density scale lengths. We also advanced both the linear and the nonlinear theory of this instability. We are thus prepared to perform experiments to address this mechanism as needed for applications.

\section{INTRODUCTION}

In recent years, laser-plasma experiments have been used to explore a number of fundamental processes in plasmas. ${ }^{1}$ This has included the radiation-driven, three-wave parametric instabilities. One of these instabilities is the Ion Acoustic Decay Insta.bility (IADI), also known as parametric decay, which involves the decay of an electromagnetic pump into an electron-plasma wave and an ion-acoustic wave. Studies of the IADI in laser plasmas may be of value for the basic understanding of nonlinear saturation, for the diagnosis of plasma temperatures near the critical density, $n_{c}$, for the understanding of preheat in laser-fusion applications, and as a source of complementary experiments to enrich the understanding of experiments in the ionosphere. The IADI has been observed and studied in a number of laser-plasma experiments. ${ }^{(2-7)}$ Additional more recent experiments are being analyzed but are not yet in print. ${ }^{2}$ The IADI also has been observed in microwave-plasma interactions, ${ }^{3-6}$ in ionospheric modification experiments, ${ }^{7,8}$ and in lower-hybrid heating of tokamaks. ${ }^{9}$ In laser plasmas, the observations most often have involved the analysis of second harmonic emission from the plasma, although some of the more recent work has utilized Thomson scattering.

The linear theory of the IADI in homogeneous plasmas was discussed in several publications through $1970,{ }^{10-14}$ and a very special case of the instability in inhomogeneous, stationary plasmas was reported in $1971 .^{15}$ In addition, it was reported in $1967,{ }^{16}$ for homogeneous plasmas, that when the plasma wave is strongly damped the IADI becomes a four-wave instability with the added participation of an upshifted, Antistokes plasma wave. Since that time, however, no further linear theory has been done. Laser-plasma experiments, which involve both subsonic and supersonic inhomogenous flows, and which are also time dependent, go far beyond the assumptions of this prior theoretical work. This has motivated recent work in this project to extend the linear theory 
to relevant conditions, ${ }^{17}$ and some of the results of this work are summarized here. Related work to understand the nonlinear states is also underway. ${ }^{18}$ In addition, the diagnosis of the IADI by second harmonic emission, and the implications of nonlinear turbulence for this emission, have not been clearly presented. We also summarize here some of the results of an examination of this issue. ${ }^{19}$

Laser-irradiated plasmas are well known to produce second-harmonic emission by several mechanisms. Such emission has been and remains the subject of extensive study as it provides evidence of a wide range of physical effects. These include (a) scattering of laser light from electron-plasma waves produced by resonance absorption, by the ion acoustic decay instability (IADI), also known as parametric decay), ${ }^{20,1}$ and by the modulational instability near the critical density; ${ }^{1}$ (b) radiation by transverse currents driven by whatever spectrum of electron plasma waves exists near the critical density; and (c) emissions from the interaction of incident and reflected laser light in filaments. ${ }^{21,22}$ In the present experiments, we measured such emission under conditions in which the Stokes feature can be produced by plasma waves and Langmuir turbulence driven by the IADI. In subsequent experiments, we used Thomson scattering to make the first observations of the plasma waves driven by acoustic decay in a warm plasma with long density scale lengths.

\section{THE ION ACOUSTIC DECAY INSTABILITY}

The three-wave IADI satisfies the usual matching conditions:

$$
\omega_{o}=\omega_{e p w}+\omega_{i a}, \quad \text { and } \quad \mathbf{k}_{o}=\mathbf{k}_{e p w}+\mathbf{k}_{i a}
$$

Here the frequencies and wavenumbers of the pump, electron-plasma, and ion-acoustic waves are designated here by $\omega_{0}, \mathbf{k}_{0}, \omega_{e p w}, \mathbf{k}_{\mathrm{epw}}$, and $\omega_{i a}, \mathbf{k}_{\mathrm{ia}}$, respectively. Throughout this paper, we will use the subscripts $i a, e p w$, and $o$ to refer to the ion-acoustic wave, the resonant plasma wave, and the pump, respectively. Figure 1 shows a typical k-matching triangle for laser-plasma conditions.

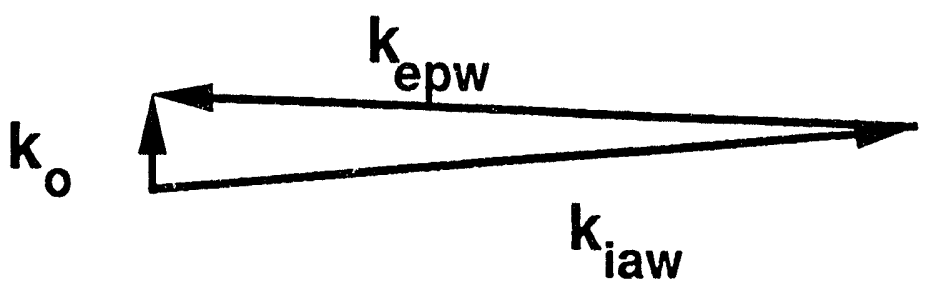

Figure 1. A typical k-matching triangle for the IADI in a laser plasma. 
Because the plasma frequency is of order $99.9 \%$ of the pump frequency, $\omega_{e p w}$ can be set equal to $\omega_{0}$ to determine $k_{e p w}$. One finds

$$
k_{e p w}=\frac{\omega_{o}}{\sqrt{3} \mathrm{v}_{t h}} \sqrt{1-\frac{n}{n_{c}}}=k_{o} \frac{c}{\sqrt{3} \mathrm{v}_{t h}}
$$

where $\mathrm{c}$ is the speed of light and $\mathrm{v}_{t h}$ is the electron thermal velocity, defined as $\left(T_{e} / m\right)^{1 / 2}$, where $T_{e}$ is the electron temperature in energy units. Thus $k_{e p w}$ (and $k_{i a}$ ) are larger at lower densities and in cooler plasmas. Note the interesting consequence, however that $k \lambda_{D}$ is independent of $T_{e}$ and depends only on density, $n$. (Here $\lambda_{D}$ is the ele

ctron Debye length.) The ratio $k_{e p w} / k_{o}$ is independent of density but is larger at lower temperatures. This is one reason why $k_{o}$ is often negligible in laboratory or ionospheric studies of the LADI. The other reason, which also may apply in laser plasmas of sufficiently-short density scale length, is that the instability may occur within the Airy pattern established near the critical density.

Figure 2 shows, in the plane of $\mathbf{k}_{0}$ and the laser electric field, $E_{L}$, the circle where the matching conditions are satisfied. The temporal growth rate, $\gamma_{0}$, of the IADI in a homogeneous plasma is proportional to $\sin \theta$, with $\theta$ as defined in the figure. Thus, the wavevector direction for which $\mathbf{k}_{\text {epw }}$ is aligned with $\mathbf{E}_{\mathrm{L}}$ corresponds to the largest temporal growth rate. This linearly fastest-growing mode dominates the development of the instability in homogeneous plasmas, even in nonlinear regimes. However, in inhomogeneous plasmas this mode often saturates at a lower amplitude that other modes. In this case, one expects the detailed behavior to be sensistive to the amplitude of the fastest-growing mode at saturation. It may or may not become large enough to affect the other modes. 
We have also found that laser plasmas are not necessarily in the 3-wave regime for the IADI. A basic description of the 4-wave instability exists, ${ }^{16}$ although we have yet to complete work on the application of the 4-wave theory to flowing, inhomogeneous plasmas. To be in the 3-wave regime, which requires that the plasma wave damping, $\gamma_{e p w}$, be less than $2 \omega_{i a}$, a laser plasma must be sufficiently warm and low $\mathrm{Z}$. We find that the 3-wave theory is valid for $\mathrm{CH}$ plasmas irradiated by a $1 \mu \mathrm{m}$ laser with temperatures of at least a few hundred $\mathrm{eV}$, for densities below about $0.95 n_{c}$.

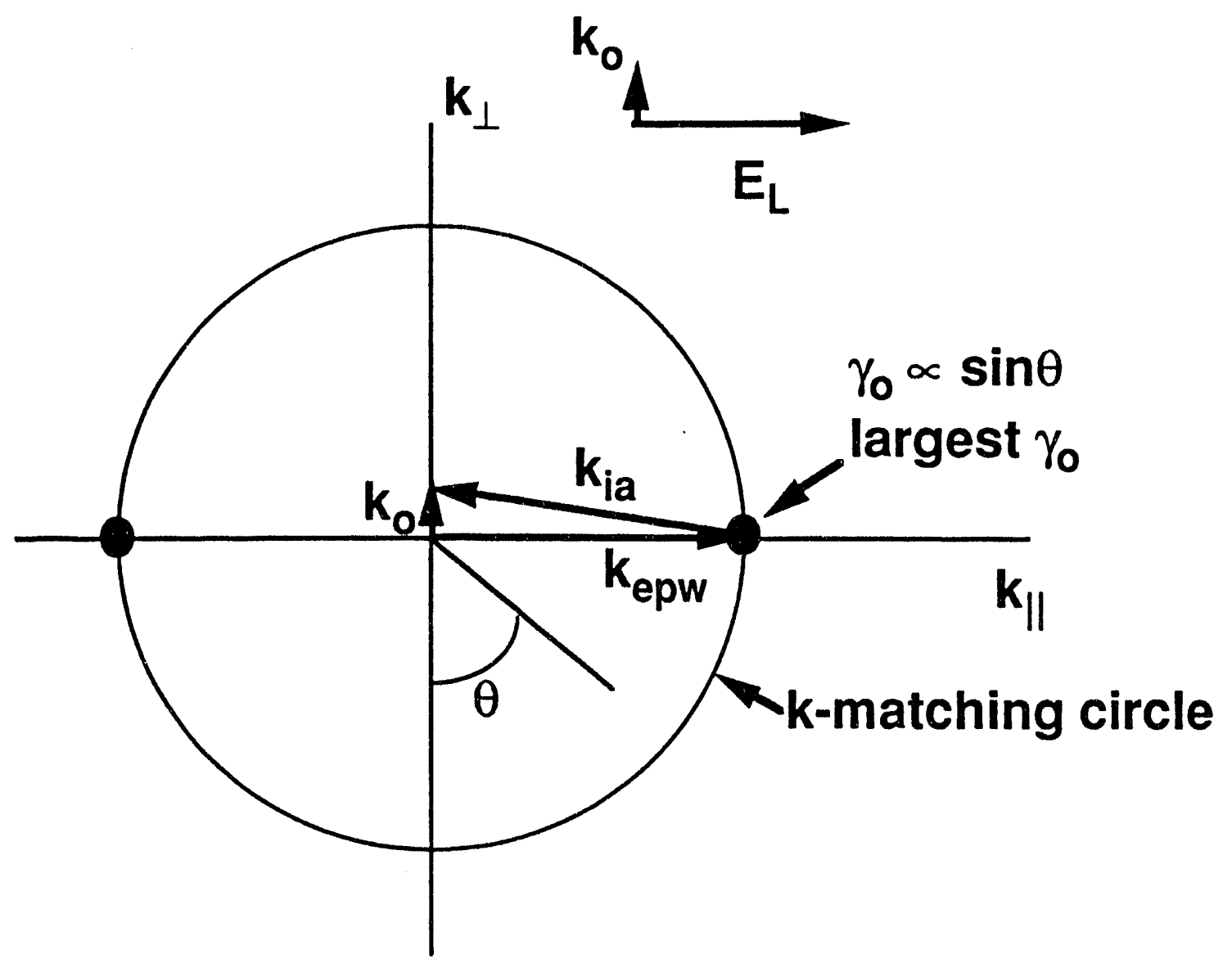

Figure 2. At a fixed density, the matching conditions imply that $k_{e p w}$ lies very nearly on a circle. The largest growth rate occurs when $k_{e p w}$ is aligned with $\mathbf{E}_{\mathrm{L}}$, as indicated by the two dots. In the IADI literature, the $\mathbf{k}_{\|}$axis is most often in this direction. 


\section{THE IADI IN INHOMOGENEOUS PLASMAS}

When the IADI is driven in an inhomogeneous plasma, several complications are introduced, as illustrated in Figure 3. In general there is a density gradient, $\nabla n$, and a flow velocity gradient, $\nabla u$. In laser plasmas these are typically opposed. As a result of these gradients, there is typically only one matching surface where plasma waves of a given frequency and trajectory are resonant for IADI. There is a finite rate of dephasing of the three waves as they propagate away from the matching surface, and this typically limits the amplification that the IADI can produce. The ion wave propagates with negligible refraction, but the plasma wave does refract and a more complicated theoretical analysis is required if the plasma wave is near its turning point. In addition, as mentioned above, the pump field may have significant spatial structure resulting from reflection at critical.

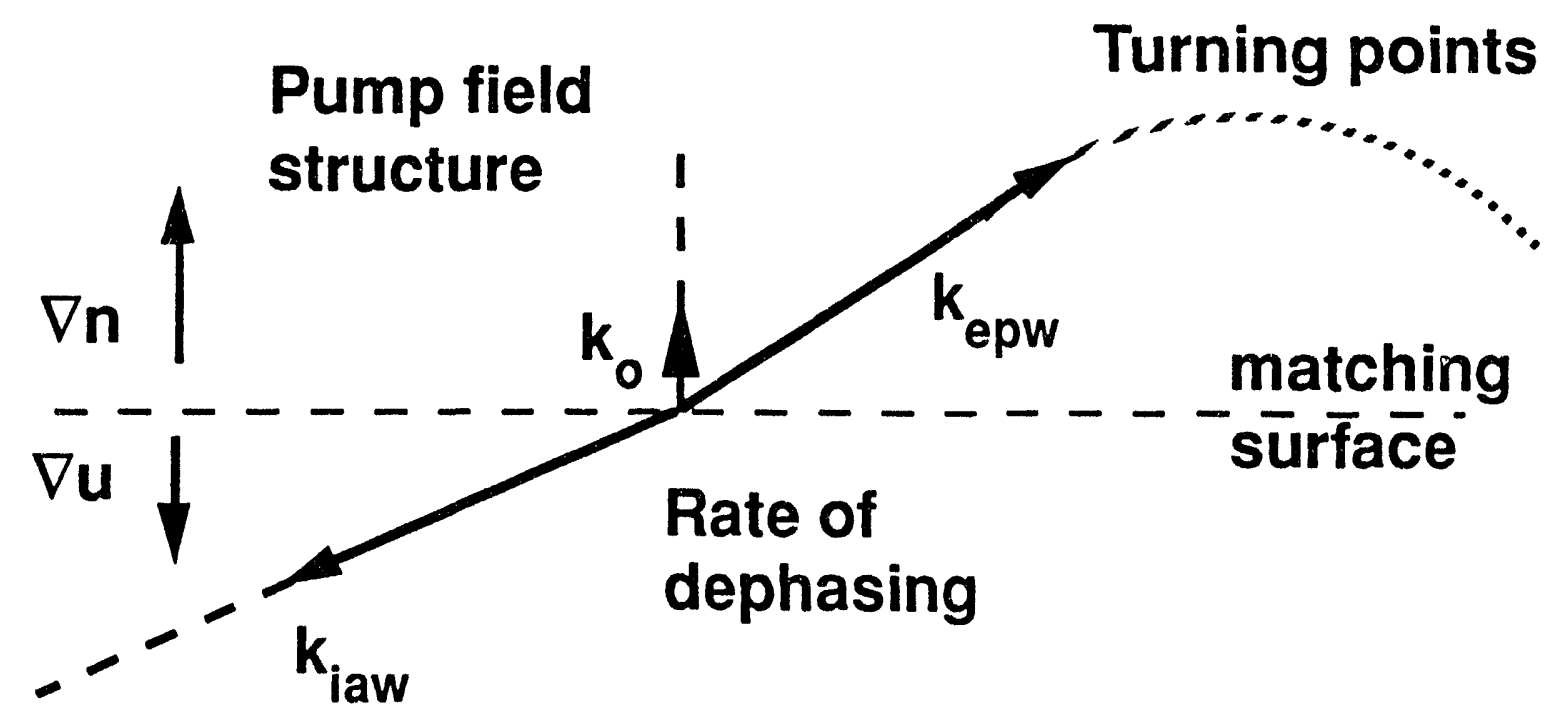

Figure 3. This figure illustrates the issues which are introduced by inhomogeneity. 
Figure 4 illustrates the interplay of these issues for a plasma wave of a given trajectory. For most plasma waves, the matching points for the IADI, A and $\mathrm{A}^{\prime}$, do not lie near the turning point TP and the IADI produces a finite amplication that can be calculated by linear WKB theory. The most-strongly-amplified waves, however, will be those for which $\mathrm{A}$ or $\mathrm{A}^{\prime}$ overlaps with a point $\mathrm{C}$ where there is no increase in dephasing as the waves propagate. The most important question for laser-plasma experiments is whether such a point $C$ exists and if so which waves also have a matching point at $C$. Such waves will be the most-strongly amplified. The prior literature ${ }^{15}$ discusses only the case for which $\nabla u=$ 0 , for which $\mathrm{C}$ is at $\mathrm{TP}$, and for which the ion wave damping is very strong.

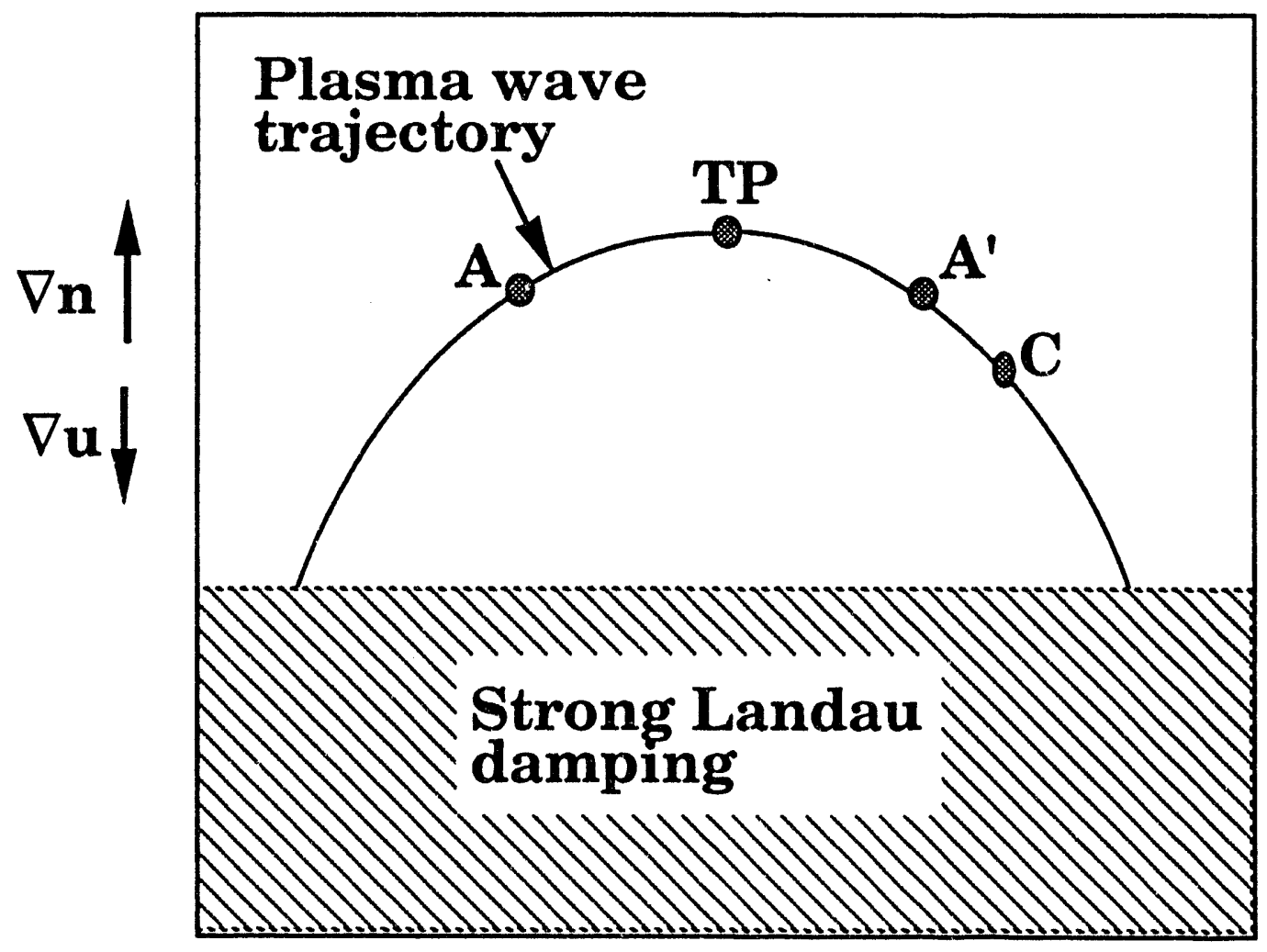

Figure 4. Plasma waves can be identified by their frequency and trajectory. A typical plasma wave will be strongly Landau damped at low density, and will propagate through a turning point TP if launched and undisturbed. Along the way, such a wave will pass through two points, $A$ and $A^{\prime}$, at which it satisfies the matching conditions for IADI. In general there may be some single point, $\mathrm{C}$, at which the dephasing rate of the three waves vanishes. 
If we let $\phi$ be the phase mismatch among the three waves, then the square of the linear dephasing rate, $B$, is given by ${ }^{23}$

$$
B=\left(\partial_{t}+\mathbf{v}_{e p w} \cdot \nabla\right)\left(\partial_{t}+\mathbf{v}_{i c} \cdot \nabla\right) \phi
$$

in which the group velocities of the plasma wave and the ion wave are $v_{e p w}$ and $v_{i a}$, respectively, and the ion-wave velocity is taken as constant. (There are slight caveats regarding $\mathrm{v}_{\mathrm{ia} .}{ }^{17}$ ) One can show that $B$ can be written as

$$
B=\mathbf{v}_{i a} \cdot \dot{\mathbf{k}}_{\text {epw }}+\mathbf{v}_{e p w} \cdot \dot{\mathbf{k}}_{i a}+\text { other terms }
$$

Here the overdot represents the convective derivative of the wave number of a wave packet, which can be related to the zeroth-order dispersion functions for wave $j, D_{j}$, as $\dot{\mathbf{k}}_{i}=\left(\partial_{t}+\mathbf{v}_{i} \bullet \nabla\right) \mathbf{k}_{i}=\partial_{\mathrm{x}} D_{i} / \partial_{\omega} D_{i}$. The important point here is that the first term of eq. 4 is dominant for IADI. It includes an important factor of $\mathbf{u} \bullet \nabla n$. In contrast, for SBS, $v_{\text {epw }}$ is replaced by $c$ and the second term of eq. 4 is dominant, with a primary contribution proportional to $\nabla u$. As a result, $\nabla u$ is the most important aspect of inhomogeneity for SBS but $\mathbf{u} \bullet \nabla n$ is most important for IADI. We also note that the quantity designated by $B$ here is usually designated as $K^{\prime} v_{1} v_{2}$ in the prior literature. However, calculations are easier and time dependence is easily included with the present formalism. ${ }^{23}$

If one keeps only the dominant term, then the dephasing rate is

$$
B=\left(\frac{-\omega_{p e}^{2} c_{s}}{2 \omega_{e p w}}\right)\left\{\left(\frac{\mathbf{u}}{c_{s}}+\hat{\mathbf{k}}_{i a}\right) \cdot\left(\frac{\nabla n}{n}\right)\right\},
$$

in which $c_{s}$ is the sound speed, $\omega_{p e}$ is the plasma frequency, and the caret designates a unit vector along $\mathbf{k}_{\mathrm{ia}}$. This has the implication, illustrated in figure 5 , that the direction of zero dephasing varies with the flow velocity. When there is no flow, then the waves along $\mathbf{k}_{\|}$ with the largest temporal growth rate are also the waves with zero dephasing. However, as the flow velocity increases the angle at which the dephasing is zero decreases and the waves with zero dephasing become increasingly parallel with the flow velocity. This is not surprising once one realizes that the flow carries the ion-wave wave packet downstream, with the implication that the plasma waves must also move downstream if they are to stay coupled to the ion waves. Actual experiments often produce supersonic flow in the IADI region. For supersonic flow there is no direction of zero spatial dephasing. The linear WKB theory, using geometric optics, indicates that the amplification is finite at all angles under such conditions. Whether richer behavior is possible for plasma waves that are near their turning point remains to be determined. 


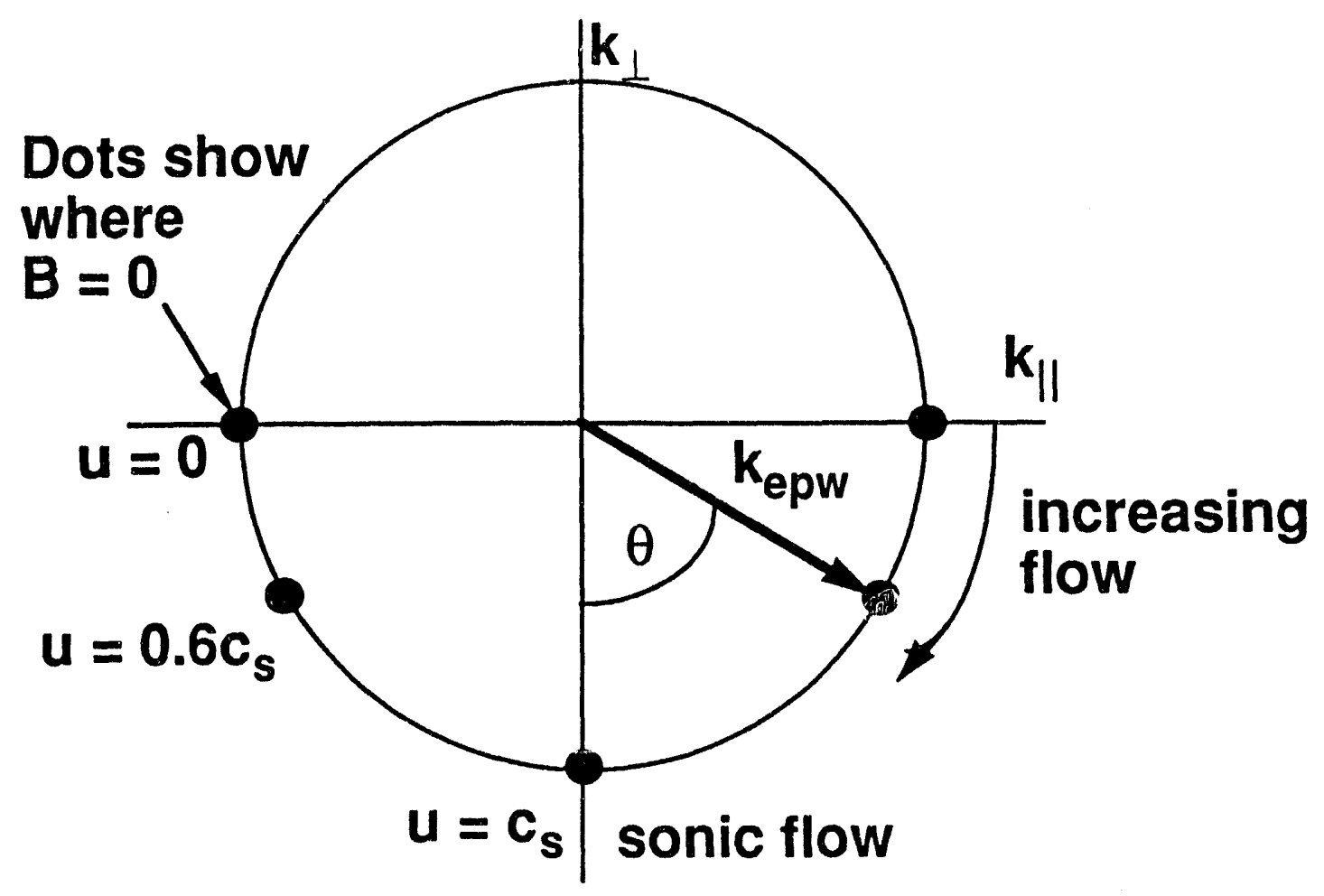

Figure 5. The direction of zero dephasing changes as the flow velocity increases, as illustrated here. For supersonic flow, there are no directions of zero dephasing.

\section{SECOND HARMONIC EMISSION}

Because of its ease of detection, second harmonic emission is very often used to diagnose phenomena near the critical density. There are two essential mechanisms by which the IADI san lead to second harmonic emission, as illustrated in fig. 6. In Thomson scattering, the laser light beats with the plasma wave, producing transverse currents that radiate at the

\section{Thomson scattering}

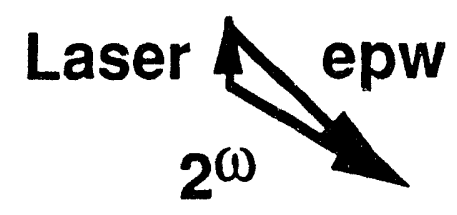

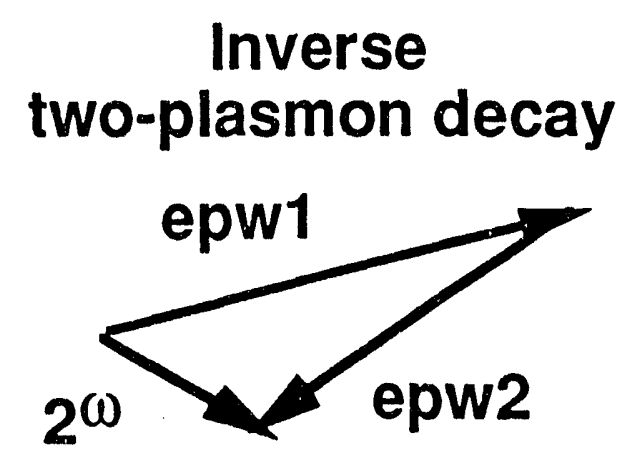

Figure 6. The twc sssential mechanisms of second harmonic production are Thomson scattering and inverse two-plasmon decay. 
second harmonic frequency. One can see from the figure that the required $\mathrm{k}_{\mathrm{epw}}$ is not much larger than the second harmonic wavenumber. This in practice implies that Thomson scattering is only important for densities very near the critical density or for emission from very cold plasmas. In laser plasmas, the implication is that Thomson scattering is unimportant for laser wavelengths $\leq 1 \mu \mathrm{m}$. Experiments have occasionally observed a narrow feature, comparatively close to the exact second harmonic frequency, that $c_{i} .1$ reasonably be attributed to Thomson scattering.

In contrast, examination of fig. 6 shows that inverse two-plasmon decay requires larger $k_{\text {epw }}$ and can accommodate a much larger range of $k_{\text {epw }}$. This implies that this mechanism is effective at comparatively lower densities. Quantitative evaluation shows that one would expect it, rather than Thomson scattering, to be responsible for the emission observed in laser plasma experiments using wavelengths $\leq 1 \mu \mathrm{m}$. In addition, it does produce frequencies that are consistent with those observed. However, there is also a problem with this mechanism as a source of the emission observed in experiments. When two plasma waves beat to produce second-harmonic currents, only the transverse currents contribute to radiation from the plasma. This has the implication that plasma waves which form isosceles triangles do not radiate. For the IADI, since the frequencies of all the plasma waves are nearly the same, such waves can only form nearly isoceles triangles (even after propagation). The slight differences in $k_{\text {epw }}$ do produce small but finite transverse currents. In contrast, small $(-10 \%)$ broadening of $k_{e p w}$ by nonlinear effects such as Langmuir turbulence can result in much larger transverse currents. Broadening of this magnitude has been observed in the nonlinear simulations mentioned above. ${ }^{18}$ Thus, it appears likely that the observed second harmonic emission occurs by inverse two-plasmon decay but involves plasma waves that have been affected by nonlinear broadening or turbulence.

\section{CONCLUSION OF THEORETICAL WORK}

Plasma flow plays a key role in the predicted linear development of the IADI in laser plasmas. Flow alters the direction of maximum predicted amplification and supersonic flow implies that the spatial dephasing rate is finite at all angles. Becuase the wavenumbers of the plasma waves driven in the warm laser plasmas produced in most modern experiments are larger than the wavenumber of second harmonic emission, inverse two-plasmon decay is the most likely source of the observed second harmonic emission. Nonlinear broadening and turbulence also probably have an important role in such emission. The results summarized here must be taken into account if one is to design, diagnose, and analyze experiments to carefully study the Ion Acoustic Decay Instability in warm, flowing, inhomogeneous laser plasmas. 


\section{THE MULTICHANNEL EXPERIMENT AND PLASMA CONDITIONS}

We used one beam of the LULI laser and varied the energy of the laser pulse, $\mathrm{E}_{\mathrm{L}}$, from less than 1 Joule to more than 80 Joules. The laser beam, of $1.053 \mu \mathrm{m}$ wavelength and $550 \pm 75$ ps gaussian pulse width, was focused onto the target through a random phase plate ( $4 \mathrm{~mm}$ square cells) by a lens having a $70 \mathrm{~cm}$ focal length. This produced a highly-structured intensity profile at the target plane, with a minimum speckle size of 18 $\mu \mathrm{m}$ and an overall Airy envelope having its first zero at $370 \mu \mathrm{m}$. This produces an effective spot size of about $190 \mu \mathrm{m}$ containing $80 \%$ of the energy, so that the average irradiating intensity (in vacuum) varied from less than $6 \times 10^{12}$ to about $5 \times 10^{14} \mathrm{~W} / \mathrm{cm}^{2}$. The laser beam irradiated $50 \mu \mathrm{m}$ thick $\mathrm{CH}$ targets at a $10^{\circ}$ angle to the target normal.

We diagnosed the second-harmonic emission using a collector array. The array used three $\mathrm{i} / 10$, spherical mirrors, located at 122,135 , and 148 degrees relative to the laser wavevector, $\mathbf{k}_{\mathbf{0}}$. Optical fibers transported the signals, with variable delay, to a spectrometer which dispersed the spectrum across the streak-camera photocathode. The data were recorded by a CCD camera. In principle one can observe second-harmonic emission either through Thomson scattering of the laser-light wave from the driven or turbulent electron-plasma waves or from the coalescence of two plasmons. In the data shown below, the Thomson-scattering process may have contrihuted to the emission at or near the exact second-harmonic frequency. However, the Stokes feature, several times 10 $\AA$ in extent, must be due to coalescence.

We performed wc-dimensional, hydrodynamic computer simulations (using LASNEX ${ }^{24}$ ) to estimate the plasma parameters for comparison with theory. The heat transport in this self-consistent, Lagrangian fluid model was modeled as Spitzer-Harm transport, limited abruptly by a flux-limiter at some specified fraction of the free-streaming heat flow. For 14 Joules of laser energy, and flux limiters of 0.03 to 0.10 , the calculated plasmas are relatively planar, with lateral scale lengths much larger than the axial scale lengths. The electron temperature is $750-950 \mathrm{eV}$, the ion temperature is $200-300 \mathrm{eV}$, and the axial scale length of the density profile at $85 \%$ of critical density is $30-60 \mu \mathrm{m}$. For these plasma conditions, standard linear theory implies that the IADI should be driven beyond the linear regime by the time of the peak of the laser pulse for the conditions of the experiment. 


\section{EXPERIMENTAL RESULTS}

Time-resoluved second-harmonic spectra at four different laser energies (all other parameters held constant) are shown in figure 7, for three angles of observation in the plane of $\mathbf{k}_{\mathbf{0}}$ and the electric field of the laser. The gross features of the spectra are independent of angle and laser intensity. The increase in observed spectral intensity with laser intensity is only slightly nonlinear. In particular, as the laser energy increased a factor of 9 , the amplitude of the second-harmonic spike increased a factor of 12 and the maximum Stokes signal increased a factor of 14 . The observations at high energy $\left(E_{L}>40 \mathrm{~J}\right)$ were quite reproducible, whereas at low energy $\left(\mathrm{E}_{\mathrm{L}}<10 \mathrm{~J}\right)$, the signal varied by up to a factor of 2 . The approximately-linear scaling of spectral intensity with $\mathrm{E}_{\mathrm{L}}$ would suggests that the amplitude of the density fluctuations producing emission through two-plasmon coalescence increased roughly proportional to $v_{0}$, while the amplitude of any fluctuations producing emission by Thomson scattering was roughly constant.

The duration and total spectral width of the observed signals increased significantly with laser energy. This is as expected since the threshold is exceeded for a longer period of time, and the IADI (the source of the turbulence) is driven more strongly at higher intensity. The full width at half maximum of the Stokes spectrum increased only slightly, however, as $\mathrm{E}_{\mathrm{L}}$ increased. This indicates that the density-dependence of the instability, and the properties of the subsequent turbulence, remained in a similar regime.

The measurements just discussed, and others made normal to the plane of the electric field of the laser, showed similar qualitative spectra in all the directions measured. Some observed trends were consistent with the linear-theory analysis. The Stokes emission began sooner for larger laser energies and in directions of lower IADI threshold (for the plasma-waves involved in the emission). However, the scaling of the observed spectral intensity with laser energy and the structure and full width of the Stokes spectrum require a nonlinear theory.

Figure 7. The development of the Stokes spectrum as laser energy increases. (a) $\mathrm{E}_{\mathrm{L}}=8.5 \mathrm{~J}, \mathrm{I}_{\mathrm{L}}=5 \times 10^{13} \mathrm{~W} / \mathrm{cm}^{2} ;$ (b) $\mathrm{E}_{\mathrm{L}}=16.5 \mathrm{~J}, \mathrm{I}_{\mathrm{L}}=1 \times 10^{14} \mathrm{~W} / \mathrm{cm}^{2}$; (c) $\mathrm{E}_{\mathrm{L}}=28 \mathrm{~J}, \mathrm{I}_{\mathrm{L}}=1.7 \times 10^{14} \mathrm{~W} / \mathrm{cm}^{2}$; (d) $\mathrm{E}_{\mathrm{L}}=71 \mathrm{~J}, \mathrm{I}_{\mathrm{L}}=4 \times 10^{14} \mathrm{~W} / \mathrm{cm}^{2}$. Each plot, from top to bottom, shows data collected at $148^{\circ}, 135^{\circ}$, and when available $122^{\circ}$ relative to $\mathbf{k}_{\mathbf{0}}$, in the plane of the laser electric field. 


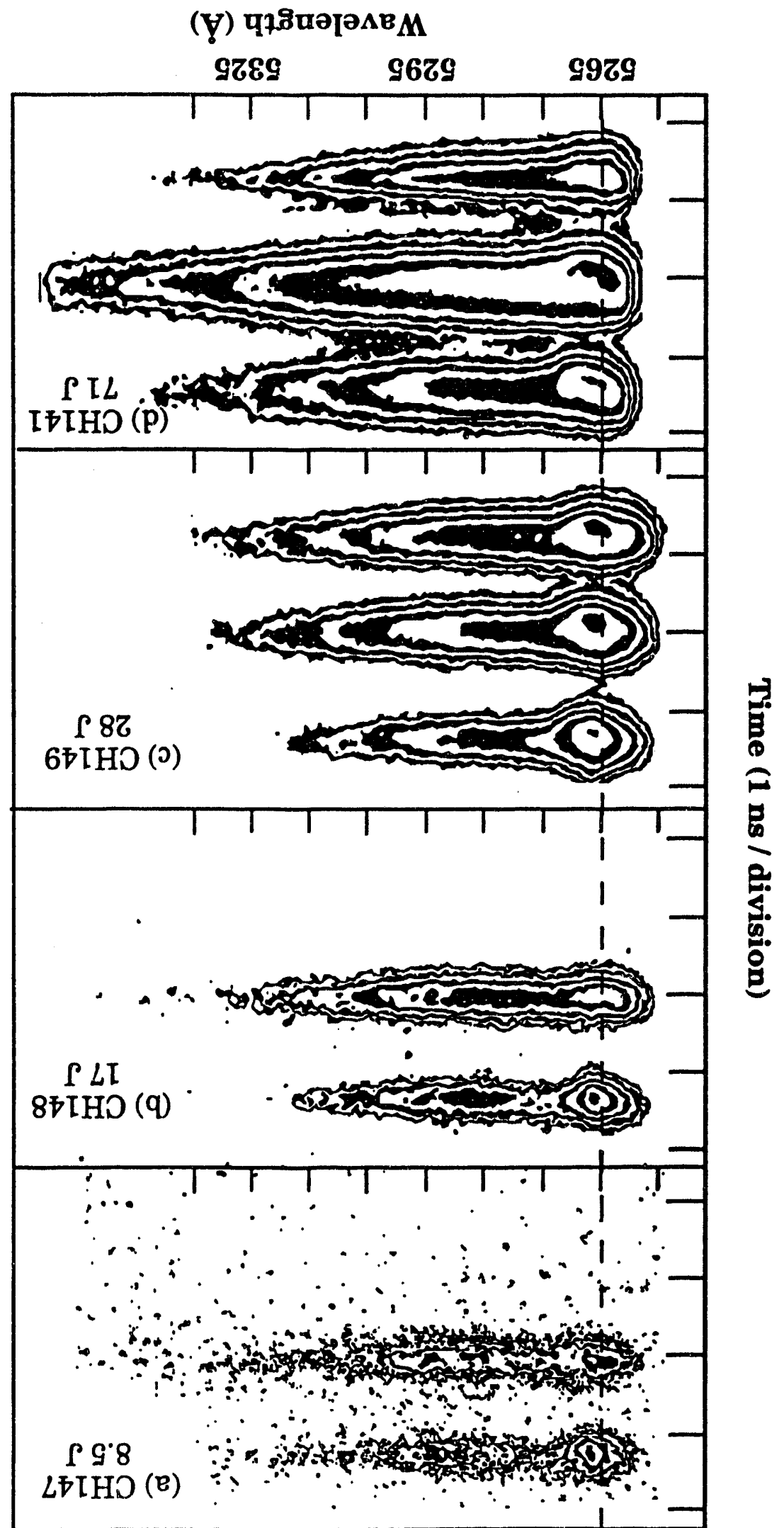

L 8. 


\section{THOMSON SCATTERING EXPERIMENTS}

During FY93 we extended our studies to larger plasmas with gentler density gradients. We used the Omega laser in collaboration with Katsu Mizuno and others of U.C. Davis, and with Wolf Seka and othurs of the University of Rochester. Several laser beams of $0.35 \mu \mathrm{m}$ wavelength produced plasmas several mm in extent with density gradients $1 / 10$ as steep as those studied previously. We then irradiated this plasma with another laser beam, of $1 \mu \mathrm{m}$ wavelength, to drive the Ion Acoustic Decay instability.

We used two diagnostic techniques. The first was to measure second harmonic emission from the unstable plasma. The electron plasma waves and subsequent turbulence drive currents that radiate at frequencies near twice that of the incident laser light, which we collected and directed through a spectrometer onto a streak camera. The spectrum we observed from these very large plasmas was similar to that observed from smaller plasmas. This supports our previous interpretation that local phenomena, not strongly sensitive to the density gradient, have been responsible for the shape of the spectrum. The second diagnostic technique was to directly detect selected electron plasma waves by Thomson scattering. We used a $0.35 \mu \mathrm{m}$ probe beam and measured the spectrum of the light scattered at roughly four times the frequency of the $1 \mu \mathrm{m}$ laser beam that drives the instability. Figure 8 shows some of the data that we are now analyzing.

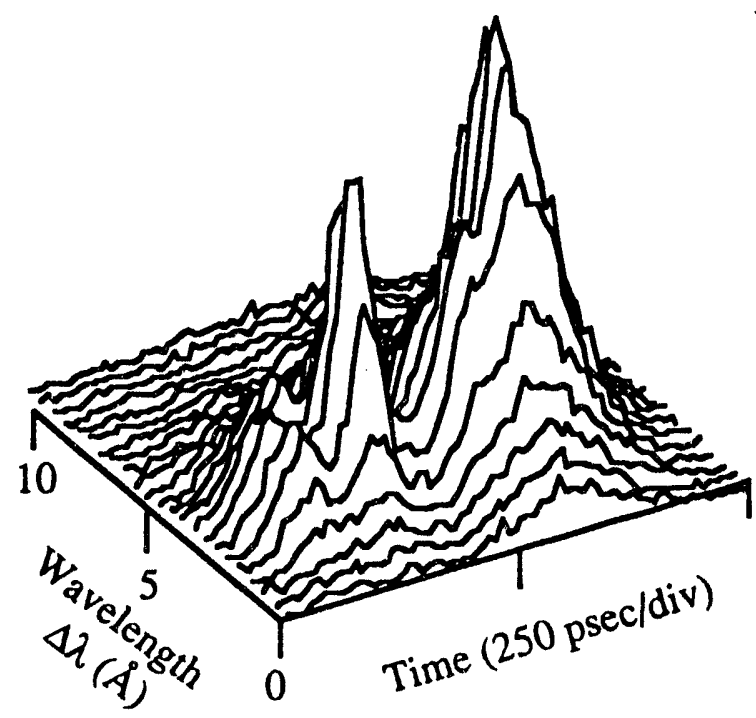

Figure 8. The spectral intensity of the Thomson-scattering from an electron plasma wave with a chosen direction of propagation is shown as a function of wavelength and time. The spectrum shows the central frequency and bandwidth of such plasma waves. 


\section{ACKNOWLEDGEMENTS}

This work was partially performed under the auspices of the U.S. Department of Energy by the Lawrence Livermore National Laboratory under Contract No. W-7405-Eng-48.

\section{PUBLICATIONS:}

1. R.P. Drake, "Three-Wave Parametric Instabilities in Long-Scale-Length, Somewhat-Planar, Laser-Produced Plasmas," Las. Part. Beams 10, 599-616 (1992).

2. B. La Fontaine, D.M. Villeneuve, H.A. Baldis, R.P. Drake, and K. Estabrook, "A test of the Landau cutoff of stimulated Raman scattering spectra as an electrontemperature diagnostic in laser-produced plasmas," Phys. Rev. Lett. 68, 484-487 (1992).

3. D.M. Villeneuve, K.L. Baker, R.P. Drake, B. Sleaford, and B.L. Fontaine, "Observation of Plasma Waves by Thomson Scattering: Saturation of Stimulated Raman Scattering," Phys. Rev. Lett. 71, 368-371 (1993).

4. $\quad$ R.P. Drake, "The Ion Acoustic Decay Instability in laser plasmas: fluid theory and second harmonic emission", in Laser Interaction and Related Plasma Phenomena (eds. Miley, G.H. \& Hora, H.), vol. 11, in press (AIP Conference Proceedings, 1994).

5. R.P. Drake, M.V. Goldman, and J.S.D. Groot, "Linear WKB theory of the radiation-driven Ion Acoustic Decay Instability in flowing, inhomogeneous plasmas", Phys. Plasmas submitted, (1994).

6. M.V. Goldman, D.L. Newman, D. Russell, D.F. DuBois, H. Rose, R.P. Drake, and A.V. Rubenchik, "New Regimes of Radiation-Driven Langmuir Turbulence Near the Decay-Instability Threshold", Phys. Plasmas in preparation, (1994).

7. R.P. Drake, B. Bauer, K.L. Baker, K. Mizuno, B. Sleaford, A.M. Rubenchik, Kent Estabrook, C. Labaune, S. Baton, T. Jalinaud, E. Schifano, M.V. Goldman and D. Newman, "Measurements of the angular structure of second harmonic emission from laser-produced plasmas", in preparation, (1994).

Several other publications are in preparation in consequence of this project. 


\section{REFERENCES}

1. W.L. Kruer, The Physics of Laser Plasma Interactions (Addison-Wesley Publishing Company, Inc., Redwood City, California, 1988).

2. K. Mizuno (private communication).

3. R.A. Stern and N. Tozar, Phys. Rev. Lett. 17, 903 (1966).

4. T.K. Chu and H.W. Hendel, Phys. Rev. Lett. 29, 634 (1972).

5. H. Dreicer, R.F. Ellis, and J.C. Ingraham, Phys. Rev. Lett. 31, 426 (1973).

6. K. Mizuno and J.S. DeGroot, Phys. Rev. Lett. 35, 219 (1975).

7. J.A. Fejer, Rev. Geophys. Space Phys. 17, 135 (1979).

8. D.F. DuBois, H.A. Rose, and D. Russell, Phys. Rev. Lett. 66, 1970 (1991).

9. Y. Takase, R.L. Watterson, M. Porkolab, C.L. Fiore, R.E. Slusher, and C.M. Surko, Phys. Rev. Lett. 53, 274 (1984).

10. D.F. DuBois and M.V. Goldman, Phys. Rev. Lett. 14, 544 (1965).

11. V.P. Silin, Sov. Phys. JETP 21, 1127 (1965).

12. E.A. Jackson, Phys. Rev. 153, 235 (1967).

13. K. Nishikawa, J. Phys. Soc. Japan 24, 916 (1968).

14. J.R. Sanmartin, Phys. Fluids 13, 1533 (1970).

15. F.W. Perkins and J. Flick, Phys. Fluids 14, 2012 ( ., 71$).$

16. D.F. DuBois and M.V. Goldman, Phys. Rev. Lett. 19, 1105 (1967).

17. R.P. Drake, M.V. Goldman, and J.S.D. Groot, Phys. Plasmas submitted, (1994).

18. M.V. Goldman, D.L. Newman, D. Russell, D.F. DuBois, H. Rose, R.P. Drake, and A.V. Rubenchik, Phys. Plasmas submitted, (1994).

19. R.P. Drake, M.V. Goldman, J.S.D. Groot, and e. al., Phys. Plasmas in preparation, (1994).

20. K. Tanaka, W. Seka, L.M. Goldman, M.C. Richardson, R.W. Short, J.M. Soures, and E.A. Williams, Phys. Fluids 27, 2187 (1984).

21. J.A. Stamper, R.H. Lehmberg, A. Schmitt, M.J. Herbst, F.C. Young, J.H. Gardner, and S.P. Obenschain, Phys. Fluids 28, 2563 (1985).

22. A. Giulietti, D. Giulietti, D. Batani, V. Biancalana, L. Gizzi, L. Nocera, and E. Schifano, Phys. Rev. Lett. 63, 524 (1989).

23. R.P. Drake and E.A. Williams, Phys. Rev. Lett. 67, 2477 (1991).

24. G.B. Zimmerman and W.L. Kruer, Comments Plasma Phys. Controlled Fusion 2, 51 (1975). 

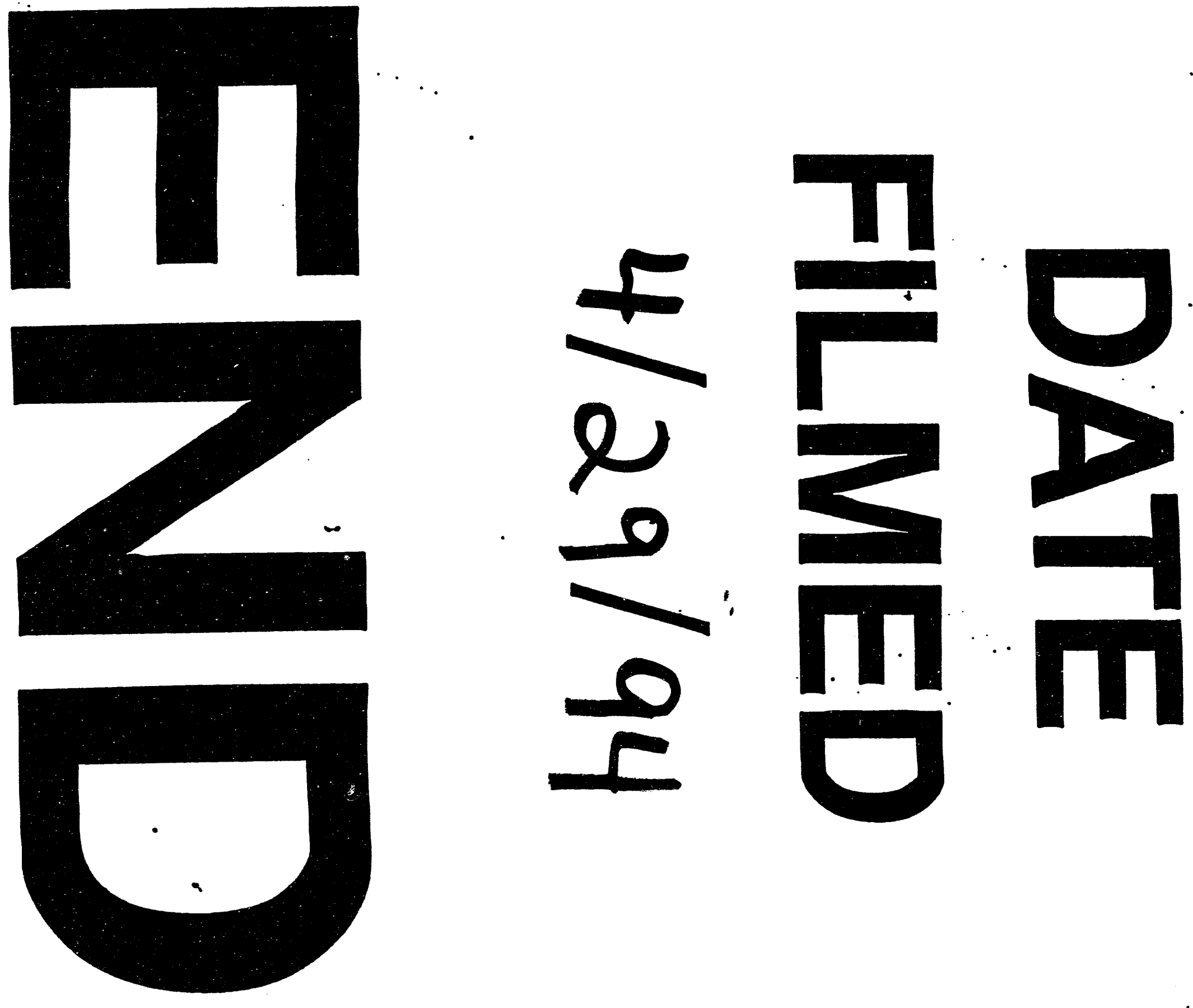
